\title{
Utility of Visual and Auditory Warning Alerting for Traffic Avoidance During UAS Operations
}

Kevin Monk, Conrad Rorie, and Zachary Roberts

This presentation covers the results of a human-in-the-loop simulation that examined the effect of caution versus warning-level alerting in the context of Unmanned Aircraft System (UAS) operations in civil airspace. Minimum Operational Performance Standards (MOPS) are currently being developed by RTCA Special Committee 228 for UAS Detect-and-Avoid (DAA) systems. Input from subject matter experts and multiple research studies have informed display requirements for DAA systems aimed at supporting timely and appropriate pilot responses to collision hazards. DAA alerting is designed to inform pilots of potential threats to DAA "well clear"; the two highest alert levels - caution and warning - indicate how soon pilot action is required and whether there is adequate time to coordinate with the air traffic controller (ATC). Additional empirical support is needed to clarify the extent to which warning-level alerting impacts DAA task performance. The present study explores the differential effects of the auditory and visual cues provided by the DAA Warning alert, and performance implications compared to caution-only alerting are discussed. 


\section{1 \\ Unmanned Aircraft Systems (UAS) Integration in the National Airspace System (NAS) Project}

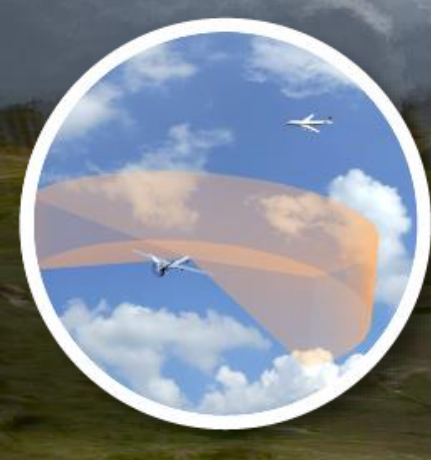

Utility of Visual and Auditory Warning Alerting for Traffic Avoidance During UAS Operations

Presented to HFES 2018:

PP1 Visual Perception and Safety:

Perception and Performance

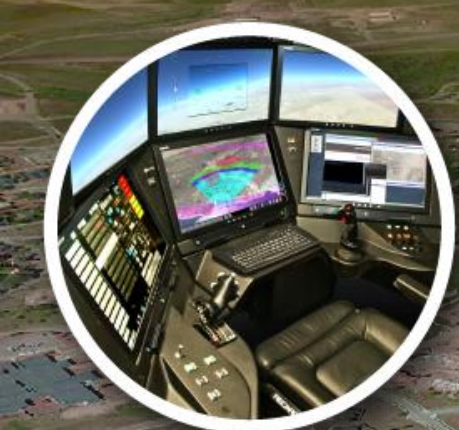




\section{Background}

- RTCA Special Committee 228 is developing the minimum operational performance standards (MOPS) for Unmanned Aircraft System (UAS) Detect and Avoid (DAA) systems operating in civil airspace

- Phase 1 of the UAS DAA MOPS prescribed minimum alerting and guidance requirements to aid pilots in avoiding a loss of DAA well clear (LoDWC)

- CFR14 Part 91 requires pilots in civil airspace to proactively maintain 'well clear' so as to avoid potential collision hazards

- A 'detect and avoid' can help compensate for pilots' removal from aircraft

- Both caution and warning-level DAA alerts were included as part of the MOPS alerting structure

- Corrective DAA Alert: caution-level; immediate awareness is required

- Pilot expected to coordinate response with ATC then maneuver

- Warning DAA Alert: warning-level; immediate maneuver is required

- Pilot expected to maneuver immediately and then contact ATC

*Consistent FAA Advisory Circular 25.1322-1 


\section{Background}

- SC228 members demonstrated frequent hesitation to included a warninglevel alert within the DAA alerting structure

- Theses members preferred to retain the warning alert for Collision Avoidance (as opposed to DAA)

- No studies directly assessed the utility of the warning-level DAA alert

- It was shown to be effective in human-in-the-loop simulations where it was included but was not systematically studied

- Experiment Objective: specifically measure the impact of the DAA warning alert on pilots' performance

- Evaluate whether the DAA Warning symbol and/or aural improves pilots' ability to remain well clear

- Systematically vary the timing of the alert to see if when an intruder registers as an alert impacts effectiveness of alert type

- Measure performance as response times and ability to maintain DAA well clear (DWC) 


\section{Experimental Design}

- Independent Variable:

- DAA Warning alert option (Between-Subjects)

- D1: No DAA Warning Alert (Corrective/Caution Alerting Only)

- D2: DAA Warning Aural Only (Retain Corrective DAA symbol)

- D3: DAA Warning Alert (Aural + Symbol)

- Embedded Variable

- Use Cases: varied by how close to a loss of DAA well clear (LoDWC) the intruder was when it first appeared

- A: $15 \mathrm{sec}$

- B: $25 \mathrm{sec}$

$--------\longleftarrow$ Warning Alert Transition

- C: $35 \mathrm{sec}$

- D: $45 \mathrm{sec} \leftarrow$ Intruder appeared first as Corrective/Caution-level alert

- E: $55 \mathrm{sec}$ 


\section{Alerting Logic}

\begin{tabular}{|c|c|c|}
\hline \multicolumn{3}{|c|}{ D1: Caution Only } \\
\hline Symbol & Name & $\begin{array}{c}\text { Aural Alert } \\
\text { Verbiage }\end{array}$ \\
\hline N/A & N/A & N/A \\
\hline & $\begin{array}{c}\text { Corrective DAA } \\
\text { Caution Alert }\end{array}$ & $\begin{array}{l}\text { "Traffic, } \\
\text { Avoid" }\end{array}$ \\
\hline & $\begin{array}{c}\text { *Preventive DAA } \\
\text { Alert }\end{array}$ & $\begin{array}{l}\text { "Traffic, } \\
\text { Monitor" }\end{array}$ \\
\hline & Guidance Traffic & $\mathrm{N} / \mathrm{A}$ \\
\hline & None (Target) & N/A \\
\hline
\end{tabular}

\begin{tabular}{|c|c|c|}
\hline \multicolumn{3}{|c|}{ D2: Warning Aural } \\
\hline Symbol & Name & $\begin{array}{c}\text { Aural Alert } \\
\text { Verbiage }\end{array}$ \\
\hline & $\begin{array}{c}\text { DAA 'Maneuver' } \\
\text { Alert } \\
\text { "Traffic, } \\
\text { Maneuver } \\
\text { Now" x2 }\end{array}$ \\
\hline & $\begin{array}{c}\text { Corrective DAA } \\
\text { Caution Alert }\end{array}$ & $\begin{array}{c}\text { "Traffic, } \\
\text { Avoid" }\end{array}$ \\
\hline & $\begin{array}{c}\text { *Preventive DAA } \\
\text { Alert }\end{array}$ & $\begin{array}{c}\text { "Traffic, } \\
\text { Monitor" }\end{array}$ \\
\hline & Guidance Traffic & N/A \\
\hline
\end{tabular}

\begin{tabular}{|c|c|c|}
\hline \multicolumn{3}{|c|}{ D3: Warning Aural + Symbol } \\
\hline Symbol & Name & $\begin{array}{c}\text { Aural Alert } \\
\text { Verbiage }\end{array}$ \\
\hline & $\begin{array}{c}\text { DAA Warning } \\
\text { Alert } \\
\text { "Traffic, } \\
\text { Maneuver } \\
\text { Now" x2 }\end{array}$ \\
\hline Corrective DAA \\
Caution Alert \\
\hline "Traffic, \\
Avoid"
\end{tabular}




\section{Test Setup}

- 15 participants

- 5 per alerting condition

- Background = 'manned' aviation pilots

- DAA Pilot Task

- Fly simulated MQ-9 Reaper along mission route (ZOA 40/41)

- Remain Well Clear from intruder aircraft

- Minimal deviation from mission route/altitude

- Coordinate with ATC (when necessary)

- Prioritize maneuver over contacting ATC after the onset of a DAA Warning alert

- Researcher acting as surrogate ATC from sim manager room

- Attend to secondary tasks

- Chat messages requesting status information 


\section{Hypotheses}

\section{- Research Question}

- What are the differential effects of the DAA Warning symbology and aural on pilot performance?

- Expected Outcome

- Faster response times and better task performance in conditions with DAA Warning alert compared to no DAA Warning

- Performance improves with more warning-level information

- D3 > D2 > D1

- Benefit of warning-level information most pronounced for encounters alerting near well clear threshold ( $\leq 25 \mathrm{sec}$ to LoDWC) 
RESUITS 


\section{Response Time}

- Aircraft Response Time $(A C R T)=$ time elapsed from the onset of the DAA alert and the first upload sent to the vehicle in response

- Effect of alert condition (D1 - 3) most pronounced when the intruder appeared inside the Warning threshold (i.e., within $25 \mathrm{sec}$ to loss of DAA well clear)

- D2 and D3 resulted in significantly faster response times against threats within warning threshold

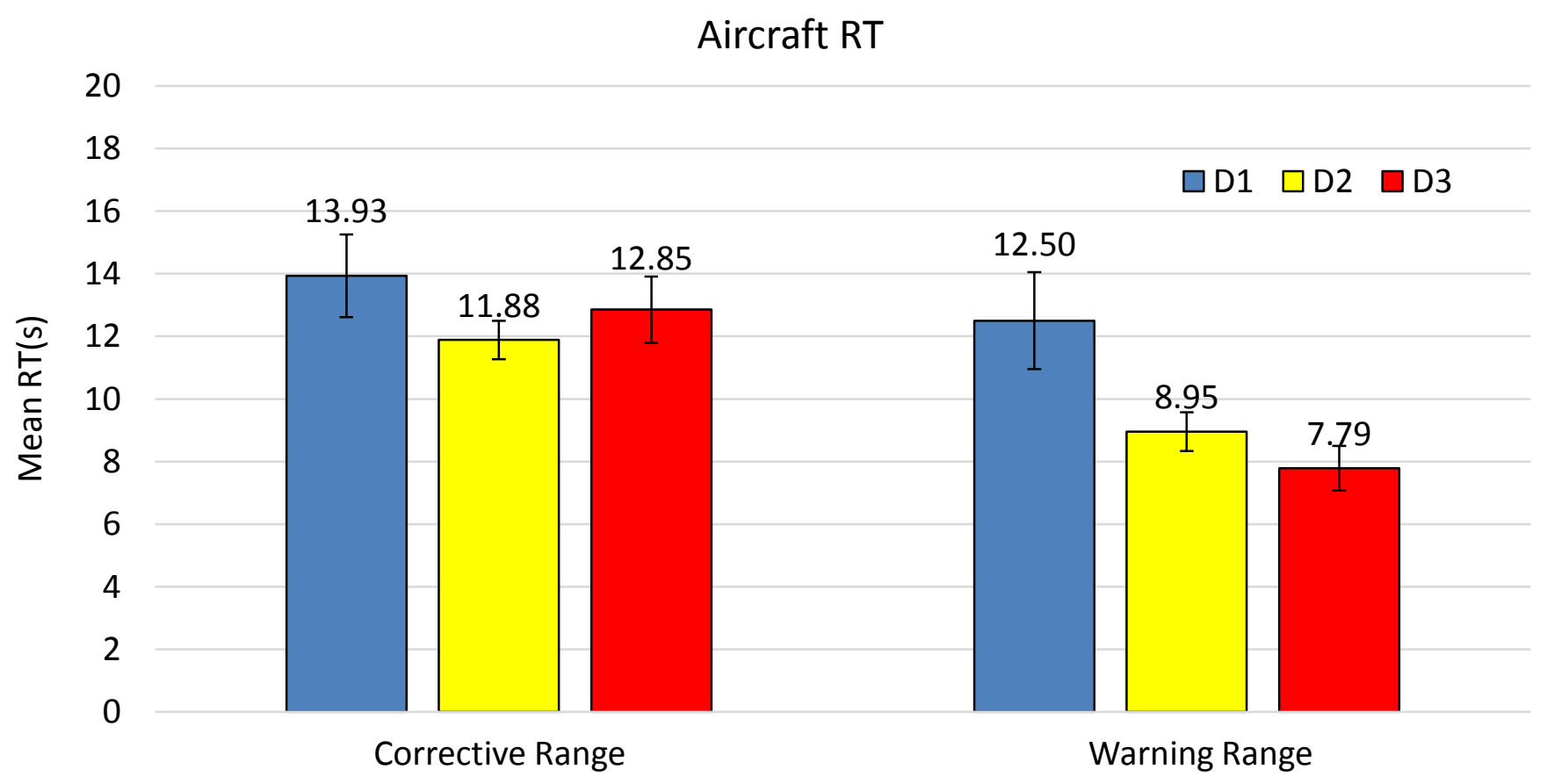

Range at First Alert 
- Pilots presented with warning-level alerting were more likely to respond appropriately to severe threats within 25s-to-LoDWC

- Pilots acted appropriately against alerts in the Corrective range

- Pilots acted most appropriately against alerts in the Warning range in the D3 condition

- Without warning-level information, pilots were much more likely to contact ATC when they should have been prioritizing maneuvering

Appropriate Pilot-ATC Coordination

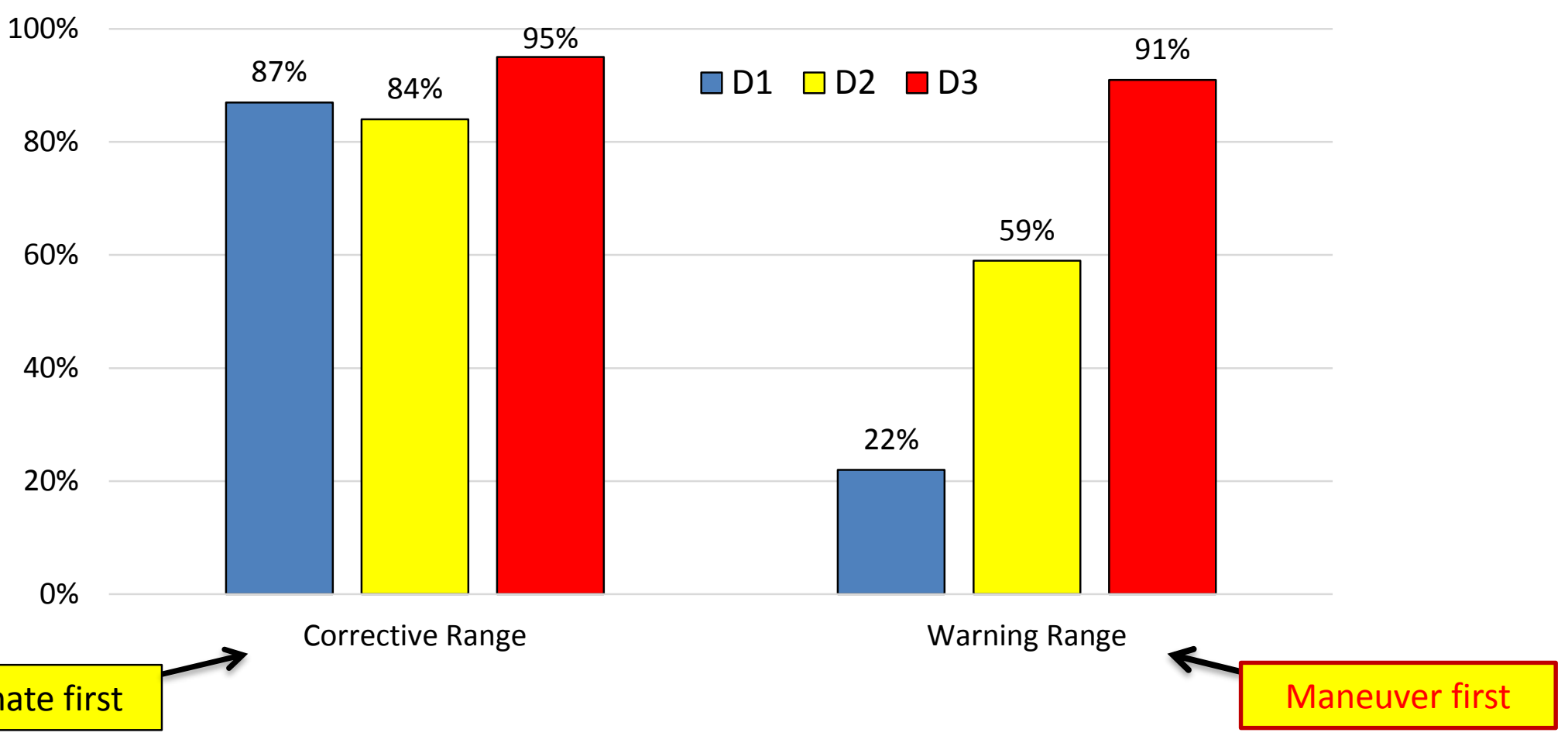




\section{Loss of DAA Well Clear}

- LoDWC Proportion

$-\mathrm{D} 1=15 \%$

$-\mathrm{D} 2=9 \%$

$-\mathrm{D} 3=2 \%$

- $91 \%$ of total LoDWC occurred in Use Cases A \& B

- None outside of 35s in any condition

- Inappropriate ATC coordination was the most common cause

- Pilots were nearly twice as likely to remain DWC against the most severe threats with the DAA Warning Alert compared to Caution-only

- Auditory Maneuver alert (D2) provided minimal benefit on its own

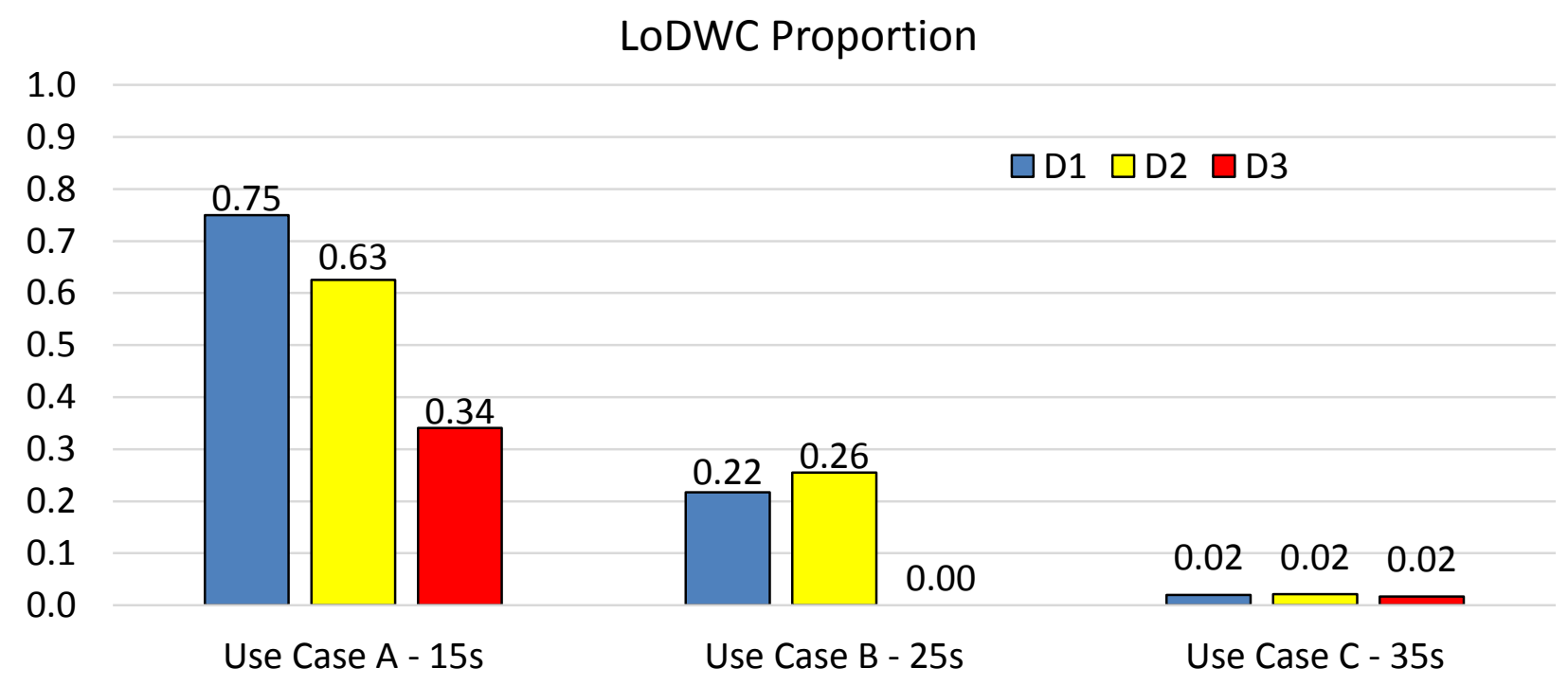


- Warning-level information improves pilot performance against severe threats (i.e., those that appear within 25 seconds to LoDWC)

- Faster response times

- Prioritized actions appropriately with indication of increased severity

- Performance remains stable at farther ranges

- Warning alerting is most conducive to DWC maintenance when auditory cue is coupled with a change in symbology

- Least pilot-responsible LoDWCs with Phase 1 MOPS DAA Warning alert

- 'Maneuver Now' aural alone did not improve separation performance compared to Caution-Only

- Potential to miss the aural change while already coordinating with ATC

- Most likely when intruder alerts at $\sim 35 s$ to LoDWC

- "Aurals start with the same word; not as attention-grabbing without distinct changes in symbology"

- "Harder to distinguish between Preventive and Corrective without no Warning symbol; trained that Red means severe" 


\section{Questions?}

conrad.rorie@nasa.gov,

kevin.j.monk@nasa.gov 